\title{
Sex and age interaction in fundamental circulatory volumetric variables at peak working capacity
}

\author{
Candela Diaz-Canestro ${ }^{1}$ and David Montero ${ }^{1,2,3^{*}}$ (B)
}

\begin{abstract}
Background: Whether the fundamental hematological and cardiac variables determining cardiorespiratory fitness and their intrinsic relationships are modulated by major constitutional factors, such as sex and age remains unresolved.

Methods: Transthoracic echocardiography, central hemodynamics and pulmonary oxygen $\left(\mathrm{O}_{2}\right)$ uptake were assessed in controlled conditions during submaximal and peak exercise (cycle ergometry) in 85 healthy young (20-44 year) and older (50-77) women and men matched by age-status and moderate-to-vigorous physical activity (MVPA) levels. Main outcomes such as peak left ventricular end-diastolic volume (LVEDV $\left.V_{\text {peak }}\right)$, stroke volume (SV peak $_{\text {, }}$, cardiac output $\left(Q_{\text {peak }}\right)$ and $\mathrm{O}_{2}$ uptake $\left(\mathrm{VO}_{2 \text { peak }}\right)$, as well as blood volume $(\mathrm{BV}), \mathrm{BV}-\mathrm{LVEDV}$ peak and LVEDV peak $-S V_{\text {peak }}$ relationships were determined with established methods.
\end{abstract}

Results: All individuals were non-smokers and non-obese, and MVPA levels were similar between sex and age groups $(P \geq 0.140)$. BV per $\mathrm{kg}$ of body weight did not differ between sexes $(P \geq 0.118)$, but was reduced with older age in men $(P=0.018)$. Key cardiac parameters normalized by body size $\left(L V E D V_{\text {peak }}, S V_{\text {peak }} Q_{\text {peak }}\right)$ were decreased in women compared with men irrespective of age $(P \leq 0.046)$. Older age per se curtailed $Q_{\text {peak }}(P \leq 0.022)$ due to lower heart rate $(P<0.001)$. In parallel, $V_{2}$ peak was reduced with older age in both sexes $(P<0.001)$. The analysis of fundamental circulatory relationships revealed that older women require a higher BV for a given $\mathrm{LVEDV}_{\text {peak }}$ than older men $(P=0.024)$.

Conclusions: Sex and age interact on the crucial circulatory relationship between total circulating BV and peak cardiac filling, with older women necessitating more BV to fill the exercising heart than age- and physical activitymatched men.

Keywords: Stress cardiac imaging, Intravascular volumes, Aerobic capacity, Female sex, Advanced age

\section{Background}

Notwithstanding the undeniable complexity of the human body, the vital organ par excellence entails a remarkable mechanistic simplicity in its most fundamental function $[1,2]$. The direct relationship between

\footnotetext{
*Correspondence: david.montero.barril@gmail.com

${ }^{2}$ Cumming School of Medicine, Libin Cardiovascular Institute of Alberta,

Faculty of Kinesiology, University of Calgary, 2500 University Drive NW, Calgary, AB, Canada

Full list of author information is available at the end of the article
}

cardiac filling and stroke volume (SV), known as the "law of the heart' or Frank-Starling mechanism, was originally formulated at the dawn of twentieth century [3]. In essence, the more the heart is filled, the greater the output $(Q=S V \times$ heart rate), until a plateau is reached $[1,2]$. In humans, experimental evidence denotes that cardiac filling in the upright position is far beneath the plateau section, such that any change in left ventricular end-diastolic volume (LVEDV) alters SV proportionally [4]. In the absence of major cardiovascular abnormalities, cardiac filling primarily depends on the filling level of the original author(s) and the source, provide a link to the Creative Commons licence, and indicate if changes were made. The images or other third party material in this article are included in the article's Creative Commons licence, unless indicated otherwise in a credit line to the material. If material is not included in the article's Creative Commons licence and your intended use is not permitted by statutory regulation or exceeds the permitted use, you will need to obtain permission directly from the copyright holder. To view a copy of this licence, visit http://creativecommons.org/licenses/by/4.0/. The Creative Commons Public Domain Dedication waiver (http://creativeco mmons.org/publicdomain/zero/1.0/) applies to the data made available in this article, unless otherwise stated in a credit line to the data. 
circulatory system, i.e., blood volume (BV) [5]. BV modulates cardiac function at rest as well as at peak exercise, in which peak $\mathrm{SV}\left(\mathrm{SV}_{\text {peak }}\right)$ exclusively determines cardiac output $\left(Q_{\text {peak }}\right)$, since peak heart rate $\left(\mathrm{HR}_{\text {peak }}\right)$ is essentially uniform for a given age [6]. Importantly, outstanding levels of cardiorespiratory fitness, as represented by $Q_{\text {peak }}$ and its pulmonary correlate peak oxygen uptake $\left(\mathrm{VO}_{2 \text { peak }}\right)$, are strongly associated with large reductions in cardiovascular and overall mortality $[7,8]$. Invariably, high levels of cardiorespiratory fitness are accompanied by BV expansion $[9,10]$. Conversely, blood withdrawal induces proportional decrements in $Q_{\text {peak }}$ and $\mathrm{VO}_{2 \text { peak }}$, which do not recover until intravasscular volumes are restored [10-12], firmly supporting the crucial role of BV in the human circulatory system.

The essential physiology of the circulatory system appears to be fairly straightforward. Yet, the fact that our current understanding is based on animal experiments and human studies mainly comprising healthy young men cannot be overlooked [13-15]. Moreover, the remarkable cardiovascular phenotypic adaptability to abnormal hemodynamic conditions, lifestyle stimuli as well as constitutional factors should be considered [16-19]. Most notably, whether the constitutional factors per excellence, i.e., sex and age, alter the fundamental circulatory relationships remains unresolved $[16,19,20]$. As a case in point, the inexorable age-dependent stiffening and remodeling of the cardiovascular system may interact with sex-specific hemodynamic and hematological characteristics $[16,17,21-23]$, plausibly altering the regulation of and relationship between primary volumetric variables in the systemic circulation [18, 24, 25]. While the prevailing emphasis on molecular cardiology is an ineluctable consequence of technological prowess, sound integrative bases of cardiovascular physiology have yet to be elucidated in a large fraction of the human population.

The purpose of the present study was to comprehensively assess the impact of sex and age on the primary physiological determinants of cardiorespiratory fitness and their intrinsic circulatory relationships. To this end, transthoracic echocardiography, central hemodynamics, pulmonary $\mathrm{O}_{2}$ uptake at rest and during submaximal and peak exercise, as well as intravascular volumes were assessed in healthy young and older women and men matched by age group and physical activity levels.

\section{Patients and methods}

\section{Study participants}

Eighty-five adult women and men matched by age group (young: $\leq 44$ years; older: $\geq 50$ years) and moderate-tovigorous physical activity (MVPA) levels were recruited via advertisements on community notice boards in the city of Calgary. Inclusion criteria comprised healthy status, absence of current medical symptoms or medication limiting incremental exercise testing, and no history of cardiac, pulmonary or neuromuscular diseases. Individuals fulfilling the above criteria but having donated blood within 3 months prior to the study were excluded. The study was approved by the Conjoint Health Research Ethics Board (REB18-1654) of the University of Calgary and conducted in accordance with the declaration of Helsinki. Prior to the start of the experiments, informed oral and written consents were obtained from all participants.

\section{Experimental design}

Participants were required to report once to our laboratory for testing in addition to a voluntary familiarization visit. All individuals were instructed to avoid strenuous exercise, alcohol and caffeine from $24 \mathrm{~h}$ prior to testing, as well as to maintain their usual baseline activity and daily dietary habits throughout the study. All measurements were performed after a fasting period $(\geq 5 \mathrm{~h})$ in a quiet room with controlled temperature between 22 and $23{ }^{\circ} \mathrm{C}$. Prior to testing the participants completed demographic and clinical questionnaires and rested in supine position for $20 \mathrm{~min}$ in order to stabilize cardiovascular, hemodynamic and hematological variables. MVPA levels were determined from established questionnaires as previously described [26].

\section{Experimental measures}

\section{Hemoglobin mass ( $H b_{\text {mass }}$ ) and blood volumes}

Hemoglobin mass $\left(\mathrm{Hb}_{\text {mass }}\right)$ was determined using the classic carbon monoxide (CO) rebreathing technique integrated in a semi-automated system with a very low typical error of measurement ( $\mathrm{TE} \leq 1.2 \%$ ), as previously described [10, 27, 28]. In brief, following 20 min of supine rest, $2 \mathrm{ml}$ of blood (baseline) was sampled from the median cubital vein via a $20-\mathrm{G}$ venflon (BD, USA) and analyzed immediately in duplicate for percent carboxyhemoglobin $(\% \mathrm{HbCO})$, hemoglobin $(\mathrm{Hb})$ concentration and hematocrit (Hct) (ABL80, Radiometer, Denmark). After the exercise test, individuals breathed $100 \% \mathrm{O}_{2}$ for 4 min to flush nitrogen from the airways. After closing the $\mathrm{O}_{2}$ input, a bolus of $1.5 \mathrm{ml} / \mathrm{kg}$ of $99.5 \%$ chemically pure $\mathrm{CO}$ (Air Liquide, Canada) was administrated into the breathing circuit. Individuals rebreathed this gas mixture for $10 \mathrm{~min}$. Then, an additional $2 \mathrm{ml}$ blood sample was obtained and analyzed in duplicate as aforementioned. The change in \% $\mathrm{HbCO}$ is used to calculate $\mathrm{Hb}_{\text {mass }}$, taking into account the small amount of $\mathrm{CO}$ that remains in the rebreathing circuit at the end of the procedure. Total red blood cell volume (RBCV), plasma volume (PV) and blood volume (BV) were determined from $\mathrm{Hb}_{\text {mass }}$, baseline $\mathrm{Hb}$ concentration and Hct, and normalized by body weight $[10,27,28]$. 


\section{Transthoracic echocardiography and central hemodynamics}

Apical four-chamber and two-chamber cine-loops were recorded via high-resolution ultrasound (Mindray Medical M9, USA) and analyzed offline (TOMTEC Imaging Systems, Royal Philips, Netherlands) at rest, at a given submaximal workload $(100 \mathrm{~W})$, and at peak heart rate $\left(\mathrm{HR}_{\text {peak }}\right)$ during incremental exercise. Following the American Society of Echocardiography and the European Association of Cardiovascular Imaging recommendations, cardiac chamber quantification was performed using the modified Simpson method (biplane method of disks) by tracing the endocardial border in both apical four-chamber and two-chamber views at end-diastole and end-systole (Additional file 1: Fig. S2) [29, 30]. Diastolic function was assessed via transmitral inflow velocities determined by pulsed-wave Doppler, with the sample volume placed between the mitral leaflet tips in the apical four-chamber view. The peak inflow velocity during early $(E)$ and late $(A)$ diastole were assessed, and the $E / A$ velocity ratio was calculated. Moreover, tissue Doppler measurements were performed in the apical four-chamber view, with a 3-mm pulsed Doppler sample volume placed at the level of septal mitral annulus and lateral tricuspid annulus. Early $\left(e^{\prime}\right)$ and late $\left(a^{\prime}\right)$ peak diastolic annular velocities, peak systolic annular velocity $\left(S^{\prime}\right)$, myocardial isovolumic contraction time (IVCT) and isovolumic relaxation time (IVRT) were determined [29, 30]. In parallel, systolic blood pressure (SBP), diastolic blood pressure (DBP) and mean blood pressure (MBP) at the heart level were continuously assessed non-invasively via Finometer PRO (Finapres Medical Systems, Netherlands), with data exported into a pre-established acquisition software (Labchart 7, AD Instruments, UK). SV was determined as LVEDV minus left ventricular end-systolic volume (LVESV), while the product of SV and HR provided cardiac output $(Q)$. Systemic vascular resistance (SVR) was calculated as the ratio of MBP and Q. Echocardiographic variables are commonly presented normalized by body surface area $\left(\mathrm{BSA}=0.007184\right.$ weight $^{0.425}$ height ${ }^{0.725}$ ) to attenuate the impact of body size on sex comparisons [31], except for intrinsic volumetric relationships within the circulatory system (assessed separately in each sex). The reproducibility of key echocardiographic and hemodynamic measurements (withinsubject coefficient of variation $(\mathrm{CV})$ ) during incremental exercise in our laboratory is $\leq 6 \%$ for left ventricular (LV) volumes and $\leq 3 \%$ for blood pressures.

\section{Aerobic capacity}

An established incremental exercise protocol [10, 27] was implemented using an electromagnetic cycle ergometer (KICKR Core trainer, Wahoo, USA) integrated within a large lower body pressure chamber (LBNP) $(165 \times 82 \times 108 \mathrm{~cm})$ designed for exercise echocardiography. The LBNP comprises an electric hydraulic jack that enables to select any degree from 0 to $45^{\circ}$ of left lateral tilting. The combination of the left semilateral body position $\left(17^{\circ}\right.$ relative to the horizontal) with lower body negative pressure allows for the simultaneous assessment of cardiac function (which requires a left lateral body position for high-quality and reproducible imaging) and aerobic capacity via the regulation of negative pressure inside the chamber $(-50 \mathrm{~mm} \mathrm{Hg})$ to induce hemodynamic loads characteristic of the upright position, a physiological requirement to achieve $\mathrm{VO}_{2 \text { peak }}[32,33]$. The test started with a warm-up period of $3 \mathrm{~min}$ at $20-30 \mathrm{~W}$ workloads. Thereafter, the workload was increased by $15-30 \mathrm{~W}$ every $60 \mathrm{~s}$ until exhaustion was reached in the recommended total duration of 7-10 min. $\mathrm{O}_{2}$ uptake and $\mathrm{CO}_{2}$ output were continuously measured and normalized by body weight (CardioCoach $\mathrm{VO}_{2}$, KORR Medical, USA). Calibration of the gas analyzers and the flowmeter was performed prior to each test. Breath-by-breath values were averaged over $15 \mathrm{~s}$. The highest breath-bybreath average value was taken as $\mathrm{VO}_{2 \text { peak }}$ provided that two of the following established criteria were fulfilled: plateau in $\mathrm{O}_{2}$ uptake despite increased workload, agepredicted $\mathrm{HR}_{\text {peak }} \pm 10 \mathrm{bpm}$ [34], respiratory exchange ratio $\geq 1.1[10,35]$.

\section{Statistical analysis}

Statistical analysis was performed using SPSS 25.0 (SPSS, Chicago, IL). Data were tested for normal distribution with the Kolmogorov-Smirnov test and for homogeneity of variances with the Levene's test. Comparisons among sex and age groups were performed via independent sample $t$ tests. In addition, ANCOVA was used to determine the effects of sex and age on fundamental volumetric relationships in the circulatory system at peak exercise: (i) BV-LVEDV $V_{\text {peak }}$ and (ii) $L V E D V_{\text {peak }}-S_{\text {peak }}$. A two-tailed $P$-value less than 0.05 was considered significant. All data are reported as mean $( \pm \mathrm{SD})$.

\section{Results}

General characteristics, resting cardiac structure and function

Main general characteristics of the study participants are shown in Table 1. All individuals were non-smokers and non-obese (body mass index $<30 \mathrm{~kg} \mathrm{~m}^{2}$ ). MVPA levels were similar between sex and age groups $(P \geq 0.140)$. As expected, women presented lower anthropometric indices (height, weight, body surface area) compared with men $(P \leq 0.006)$, irrespective of the age group. No sex effect was found regarding resting blood pressure and 
Table 1 General characteristics, resting cardiac structure and function

\begin{tabular}{|c|c|c|c|c|c|c|c|c|}
\hline & \multicolumn{3}{|l|}{ Young } & \multicolumn{3}{|l|}{ Older } & \multicolumn{2}{|c|}{$\begin{array}{l}P \text { for age } \\
\text { comparison }\end{array}$} \\
\hline & 우 & 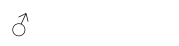 & $P$ for sex & 우 & $\delta$ & $P$ for sex & q & $\delta$ \\
\hline$n$ & 14 & 14 & & 29 & 28 & & & \\
\hline Age (year) & $33.1 \pm 6.0$ & $28.5 \pm 6.9$ & 0.070 & $64.9 \pm 7.3^{\mathrm{a}}$ & $64.9 \pm 6.4^{\mathrm{a}}$ & 0.998 & $<0.001$ & $<0.001$ \\
\hline MVPA (h) & $6.0 \pm 3.3$ & $8.5 \pm 5.2$ & 0.140 & $5.9 \pm 2.7$ & $6.2 \pm 3.1$ & 0.753 & 0.982 & 0.149 \\
\hline Height (cm) & $169.0 \pm 3.8$ & $179.4 \pm 9.9^{*}$ & 0.001 & $162.8 \pm 7.9^{\mathrm{a}}$ & $177.3 \pm 5.6^{*}$ & $<0.001$ & 0.008 & 0.378 \\
\hline Weight (kg) & $65.0 \pm 5.9$ & $78.7 \pm 15.9^{*}$ & 0.006 & $60.4 \pm 9.5$ & $80.3 \pm 9.3^{*}$ & $<0.001$ & 0.103 & 0.683 \\
\hline BMI $\left(\mathrm{kg} \mathrm{m}^{-2}\right)$ & $22.8 \pm 2.1$ & $24.3 \pm 3.6$ & 0.170 & $22.7 \pm 2.7$ & $25.6 \pm 2.8^{*}$ & $<0.001$ & 0.941 & 0.236 \\
\hline $\mathrm{BSA}\left(\mathrm{m}^{2}\right)$ & $1.74 \pm 0.08$ & $1.97 \pm 0.22^{*}$ & 0.001 & $1.64 \pm 0.15^{\mathrm{a}}$ & $1.98 \pm 0.12^{*}$ & $<0.001$ & 0.025 & 0.968 \\
\hline SBP $(\mathrm{mm} \mathrm{Hg})$ & $130.9 \pm 16.2$ & $132.8 \pm 19.3$ & 0.783 & $138.8 \pm 32.2$ & $147.2 \pm 18.2^{\mathrm{a}}$ & 0.233 & 0.393 & 0.023 \\
\hline DBP (mm Hg) & $73.6 \pm 20.6$ & $73.8 \pm 12.5$ & 0.974 & $76.9 \pm 18.5$ & $78.4 \pm 12.7$ & 0.729 & 0.599 & 0.277 \\
\hline MBP (mm Hg) & $88.0 \pm 11.5$ & $88.6 \pm 18.0$ & 0.918 & $96.2 \pm 21.1$ & $101.0 \pm 12.9^{\mathrm{a}}$ & 0.302 & 0.184 & 0.014 \\
\hline $\mathrm{HR}(\mathrm{bpm})$ & $62.1 \pm 9.2$ & $59.4 \pm 8.5$ & 0.426 & $56.8 \pm 7.2^{\mathrm{a}}$ & $55.1 \pm 5.9$ & 0.342 & 0.044 & 0.061 \\
\hline \multicolumn{9}{|l|}{ Resting echocardiography } \\
\hline $\mathrm{RA}\left(\mathrm{mL} \mathrm{m}^{-2}\right)$ & $21.00 \pm 9.51$ & $20.09 \pm 5.52$ & 0.761 & $17.94 \pm 6.20$ & $20.27 \pm 6.78$ & 0.180 & 0.211 & 0.932 \\
\hline RVEDA $\left(\mathrm{cm}^{2} \mathrm{~m}^{-2}\right)$ & $11.98 \pm 2.42$ & $11.53 \pm 1.85$ & 0.588 & $10.42 \pm 2.04^{\mathrm{a}}$ & $11.04 \pm 2.30$ & 0.284 & 0.032 & 0.491 \\
\hline $\operatorname{RV} E S A\left(\mathrm{~cm}^{2} \mathrm{~m}^{-2}\right)$ & $5.71 \pm 1.21$ & $4.90 \pm 0.97$ & 0.060 & $4.77 \pm 1.71$ & $5.22 \pm 2.16$ & 0.395 & 0.073 & 0.607 \\
\hline $\mathrm{LA}\left(\mathrm{mL} \mathrm{m} \mathrm{m}^{-2}\right)$ & $20.70 \pm 8.40$ & $21.55 \pm 6.31$ & 0.763 & $23.87 \pm 10.57$ & $22.41 \pm 9.76$ & 0.589 & 0.331 & 0.768 \\
\hline LVEDV $\left(\mathrm{mL} \mathrm{m} \mathrm{m}^{-2}\right)$ & $45.48 \pm 10.50$ & $59.34 \pm 14.22^{*}$ & 0.007 & $46.81 \pm 9.06$ & $50.06 \pm 12.74^{a}$ & 0.270 & 0.671 & 0.038 \\
\hline $\operatorname{LVESV}\left(m L m^{-2}\right)$ & $12.38 \pm 3.52$ & $18.50 \pm 6.89^{*}$ & 0.006 & $13.60 \pm 5.36$ & $15.29 \pm 6.96$ & 0.310 & 0.443 & 0.164 \\
\hline LVEF (\%) & $72.2 \pm 7.9$ & $69.1 \pm 5.2$ & 0.222 & $71.6 \pm 7.7$ & $70.4 \pm 7.5$ & 0.567 & 0.793 & 0.554 \\
\hline $\mathrm{SV}\left(\mathrm{mL} \mathrm{m}^{-2}\right)$ & $33.10 \pm 9.67$ & $40.62 \pm 8.61^{*}$ & 0.039 & $33.21 \pm 5.88$ & $34.78 \pm 7.57^{\mathrm{a}}$ & 0.385 & 0.964 & 0.030 \\
\hline$Q\left(L \min ^{-1} \mathrm{~m}^{-2}\right)$ & $2.02 \pm 0.51$ & $2.41 \pm 0.60$ & 0.075 & $1.99 \pm 0.72$ & $1.91 \pm 0.44^{\mathrm{a}}$ & 0.630 & 0.897 & 0.004 \\
\hline \multicolumn{9}{|l|}{ Diastolic function } \\
\hline Tricuspid annular $\mathrm{e}^{\prime}\left(\mathrm{cm} \mathrm{s}^{-1}\right)$ & $14.18 \pm 4.29$ & $12.10 \pm 2.66$ & 0.162 & $10.71 \pm 1.81$ & $9.11 \pm 1.74$ & 0.005 & $<0.001$ & $<0.001$ \\
\hline Tricuspid annular a' $\left(\mathrm{cm} \mathrm{s}^{-1}\right)$ & $9.41 \pm 2.06$ & $9.00 \pm 2.14$ & 0.633 & $14.08 \pm 2.51$ & $12.49 \pm 2.69$ & 0.046 & $<0.001$ & $<0.001$ \\
\hline Tricuspid annular $\mathrm{S}^{\prime}\left(\mathrm{cm} \mathrm{s}^{-1}\right)$ & $13.13 \pm 2.57$ & $12.81 \pm 1.30$ & 0.715 & $11.04 \pm 1.51$ & $11.07 \pm 2.04$ & 0.952 & 0.015 & 0.017 \\
\hline Mitral inflow $E\left(\mathrm{~cm} \mathrm{~s}^{-1}\right)$ & $79.24 \pm 12.69$ & $74.83 \pm 9.17$ & 0.301 & $62.23 \pm 12.04$ & $54.90 \pm 10.28$ & 0.019 & $<0.001$ & $<0.001$ \\
\hline Mitral inflow $\mathrm{A}\left(\mathrm{cm} \mathrm{s}^{-1}\right)$ & $52.54 \pm 10.74$ & $45.29 \pm 8.58$ & 0.059 & $65.68 \pm 12.11$ & $60.20 \pm 9.02$ & 0.065 & 0.001 & $<0.001$ \\
\hline E/A ratio & $1.55 \pm 0.30$ & $1.69 \pm 0.30$ & 0.213 & $0.957 \pm 0.142$ & $0.923 \pm 0.183$ & 0.451 & $<0.001$ & $<0.001$ \\
\hline Mitral annular e' $\left(\mathrm{cm} \mathrm{s}^{-1}\right)$ & $14.59 \pm 2.52$ & $14.17 \pm 2.91$ & 0.683 & $9.90 \pm 2.20$ & $9.02 \pm 1.82$ & 0.120 & $<0.001$ & $<0.001$ \\
\hline Mitral annular a' $\left(\mathrm{cm} \mathrm{s}^{-1}\right)$ & $8.44 \pm 1.26$ & $7.95 \pm 1.65$ & 0.393 & $11.52 \pm 2.37$ & $11.44 \pm 1.95$ & 0.886 & $<0.001$ & $<0.001$ \\
\hline Ele'ratio & $5.59 \pm 1.31$ & $5.44 \pm 1.09$ & 0.752 & $6.53 \pm 1.77$ & $6.23 \pm 1.38$ & 0.496 & 0.083 & 0.073 \\
\hline IVRT (s) & $0.120 \pm 0.173$ & $0.108 \pm 0.159$ & 0.859 & $0.074 \pm 0.012$ & $0.079 \pm 0.015$ & 0.204 & 0.402 & 0.513 \\
\hline IVCT (s) & $0.053 \pm 0.008$ & $0.059 \pm 0.010$ & 0.120 & $0.065 \pm 0.011$ & $0.061 \pm 0.011$ & 0.229 & 0.001 & 0.533 \\
\hline
\end{tabular}

Significant $P$ values $(<0.05)$ are highlighted in bold

Data are expressed as mean \pm SD

$\mathrm{BMI}$, body mass index; BSA, body surface area; DBP, diastolic blood pressure; HR, heart rate; IVCT, isovolumic contraction time; IVRT, isovolumic relaxation time; LA, left atria; LVEDV, left ventricular end-diastolic volume; LVEF, left ventricular ejection fraction; MBP, mean blood pressure; MVPA, moderate-to-vigorous physical activity; $Q$, cardiac output; RA, right atria; RV EDA, right ventricular end-diastolic area; RV ESA, right ventricular end-systolic area; SBP, systolic blood pressure; SV, stroke volume

${ }^{*} P<0.05$ compared with age-matched women

a $P<0.05$ compared with sex-matched young individuals

HR $(P \geq 0.302)$. Nonetheless, older men exhibited higher resting blood pressure $(P=0.014)$ and older women lower resting HR $(P=0.044)$ compared with younger sex-matched counterparts. Regarding the cardiac phenotype at rest, sex effects were detected in young individuals, women having smaller LV volumes and SV than men $(P \leq 0.039)$. Likewise, age effects were noted in the LV, older men presenting lower LVEDV, SV and $Q$ compared with young men $(P \leq 0.038)$. Furthermore, age per se reduced early peak diastolic inflow and annular velocities $(P<0.001)$, whereas late peak diastolic inflow and annular velocities were increased with older age 
$(P=0.001)$. Accordingly, the $E / A$ velocity ratio was lower in older individuals, irrespective of sex $(P<0.001)$. Sex effects were specifically detected in older individuals, with women presenting higher tricuspid annular velocities than men $(P \leq 0.045)$. Furthermore, the myocardial IVCT (septal) was exclusively increased with older age in women $(P=0.001)$.

\section{Blood $\mathrm{O}_{2}$ carrying capacity and intravascular volumes}

Table 2 presents key hematological determinants of cardiorespiratory fitness. According to established sex differences, blood $\mathrm{O}_{2}$ carrying capacity, as represented by $\mathrm{Hb}$ concentration and $\mathrm{Hb}_{\text {mass }}$, was markedly lower in women compared with men, irrespective of the age group $(P<0.001)$. An age effect was observed for $\mathrm{Hb}_{\text {mass }}$ per $\mathrm{kg}$ of body weight, which was reduced with older age in men $(P \leq 0.002)$. With respect to intravascular volumes, all of them (RBCV, PV, BV) were lower in absolute terms in women relative to men, in both young an older age groups $(P \leq 0.016)$. When normalized by body weight, RBCV remained lower in young and older women compared with age-matched men $(P<0.001)$, concurring with sex differences in $\mathrm{O}_{2}$ carrying capacity. With respect to the influence of age, total BV per kg of body weight was reduced in older compared with younger men $(P=0.018)$.

\section{Sex and age effects at peak exercise}

The hematological, hemodynamic, cardiac and pulmonary variables at peak incremental exercise are presented in Table 3. Similar to resting values, blood $\mathrm{O}_{2}$ carrying capacity at peak exercise was lower in young and older women compared with age-matched men
$(P<0.001)$. Peak blood pressures were similar between sexes $(P \geq 0.075)$, whereas $\mathrm{SBP}_{\text {peak }}$ and $\mathrm{MBP}_{\text {peak }}$ were higher with older versus younger age in women and men $(P \leq 0.018)$. Higher SVRpeak levels were observed with both female sex $(P \leq 0.038)$ and older age $(P \leq 0.010)$. Moreover, $\mathrm{HR}_{\text {peak }}$ was markedly reduced with older age $(P<0.001)$. As regards cardiac structure and function, LVEDV $_{\text {peak }}$, SVpeak and $Q_{\text {peak }}$ were lower in women compared with men irrespective of the age group $(P \leq 0.046)$. Furthermore, older age in women and men was associated with reduced $Q_{\text {peak }}(P \leq 0.022)$, primarily explained by lower $\mathrm{HR}_{\text {peak }}$ since $\mathrm{SV}_{\text {peak }}$ was not affected by older age $(P \geq 0.595)$. Absolute peak power output was lower in women compared with men in both age groups (206 \pm 34 vs. $308 \pm 58 \mathrm{~W}, P<0.001$ (young); $156 \pm 46$ vs. $197 \pm 46 \mathrm{~W}, P \leq 0.046$ (older)) and reduced with older age in both sex groups $(P<0.001)$. Finally, cardiorespiratory fitness, as determined by $\mathrm{VO}_{2 \text { peak }}\left(\mathrm{mL} \mathrm{min}{ }^{-1} \mathrm{~kg}^{-1}\right)$, was reduced with older age in both sexes $(P<0.001)$.

\section{Sex and age effects at a fixed submaximal workload}

Table 4 displays the hemodynamic, cardiac and pulmonary variables at a given absolute submaximal workload $(100 \mathrm{~W})$. Submaximal blood pressures did not differ between sexes $(P \geq 0.257)$. With older age, $\mathrm{SBP}_{100 \mathrm{w}}$ and $\mathrm{MBP}_{100 \mathrm{w}}$ were elevated in women and men $(P \leq 0.006)$. Likewise, $\mathrm{SVR}_{100 \mathrm{~W}}$ was augmented with older age irrespective of sex $(P \leq 0.004)$. In contrast, $\mathrm{HR}_{100 \mathrm{w}}$ was only affected by sex, with young and older women presenting higher values than age-matched men $(P \leq 0.013)$. Sex effects were detected regarding submaximal cardiac variables. Higher right atrial and ventricular dimensions

Table 2 Blood hemoglobin and intravascular volumes

\begin{tabular}{|c|c|c|c|c|c|c|c|c|}
\hline & \multicolumn{3}{|l|}{ Young } & \multicolumn{3}{|l|}{ Older } & \multicolumn{2}{|c|}{$\begin{array}{l}P \text { for age } \\
\text { comparison }\end{array}$} \\
\hline & q & $\delta$ & $P$ for sex & $q$ & $\delta$ & $P$ for sex & q & $\delta$ \\
\hline $\mathrm{Hb}_{\text {mass }}(\mathrm{g})$ & $655.8 \pm 86.6$ & $1011.9 \pm 133.8^{*}$ & $<0.001$ & $610.3 \pm 81.3^{a}$ & $923.8 \pm 116.0^{* a}$ & $<0.001$ & 0.046 & 0.033 \\
\hline $\mathrm{Hb}_{\text {mass }}\left(\mathrm{g} \mathrm{kg}^{-1}\right)$ & $10.3 \pm 1.5$ & $13.1 \pm 1.5^{*}$ & $<0.001$ & $10.2 \pm 1.2$ & $11.6 \pm 1.3^{* a}$ & $<0.001$ & 0.833 & 0.002 \\
\hline $\mathrm{Hb}\left(\mathrm{g} \mathrm{dL}^{-1}\right)$ & $13.2 \pm 1.0$ & $15.4 \pm 0.7^{*}$ & $<0.001$ & $13.3 \pm 0.6$ & $14.9 \pm 1.0^{*}$ & $<0.001$ & 0.512 & 0.111 \\
\hline $\operatorname{Hct}(\%)$ & $40.4 \pm 3.0$ & $47.2 \pm 1.9^{*}$ & $<0.001$ & $40.9 \pm 1.8$ & $45.7 \pm 3.1^{*}$ & $<0.001$ & 0.508 & 0.107 \\
\hline $\mathrm{RBCV}(\mathrm{mL})$ & $2248 \pm 295$ & $3407 \pm 450^{*}$ & $<0.001$ & $2060 \pm 274^{a}$ & $3111 \pm 387^{* a}$ & $<0.001$ & 0.046 & 0.033 \\
\hline $\operatorname{RBCV}\left(\mathrm{mL} \mathrm{kg}{ }^{-1}\right)$ & $34.8 \pm 5.2$ & $43.9 \pm 5.1^{*}$ & $<0.001$ & $34.5 \pm 4.2$ & $38.9 \pm 4.3^{* a}$ & $<0.001$ & 0.837 & 0.002 \\
\hline $\mathrm{PV}(\mathrm{mL})$ & $3332 \pm 508$ & $3810 \pm 470^{*}$ & 0.016 & $2983 \pm 418^{a}$ & $3709 \pm 530^{*}$ & $<0.001$ & 0.021 & 0.550 \\
\hline$P V\left(m L k g^{-1}\right)$ & $51.4 \pm 7.6$ & $49.3 \pm 6.9$ & 0.445 & $49.8 \pm 6.1$ & $46.4 \pm 6.1$ & 0.038 & 0.469 & 0.176 \\
\hline $\mathrm{BV}(\mathrm{mL})$ & $5580 \pm 717$ & $7216 \pm 882^{*}$ & $<0.001$ & $5043 \pm 667^{a}$ & $6820 \pm 796^{*}$ & $<0.001$ & 0.020 & 0.150 \\
\hline $\mathrm{BV}\left(\mathrm{mL} \mathrm{kg}{ }^{-1}\right)$ & $86.2 \pm 11.5$ & $93.2 \pm 11.5$ & 0.118 & $84.3 \pm 9.8$ & $85.4 \pm 8.8^{a}$ & 0.675 & 0.582 & 0.018 \\
\hline
\end{tabular}

Significant $P$ values $(<0.05)$ are highlighted in bold

Data are expressed as mean \pm SD

$\mathrm{BV}$, blood volume; $\mathrm{Hb}$, hemoglobin concentration; $\mathrm{Hb}_{\text {mass }}$, total circulating hemoglobin mass; $\mathrm{Hct}$, hematocrit; $\mathrm{PV}$, plasma volume; $\mathrm{RBCV}$, red blood cell volume * $P<0.05$ compared with age-matched women

${ }^{\text {a }} P<0.05$ compared with sex-matched young individuals 
Table 3 Hematological, hemodynamic, cardiac and pulmonary variables at peak exercise

\begin{tabular}{|c|c|c|c|c|c|c|c|c|}
\hline & \multicolumn{3}{|l|}{ Young } & \multicolumn{3}{|l|}{ Older } & \multicolumn{2}{|c|}{$\begin{array}{l}P \text { for age } \\
\text { comparison }\end{array}$} \\
\hline & q & $\hat{o}$ & $P$ for sex & q & $\hat{\sigma}$ & $P$ for sex & q & $\hat{\sigma}$ \\
\hline \multicolumn{9}{|c|}{ Peak blood O2 carrying capacity } \\
\hline$H b_{\text {peak }}\left(\mathrm{g} \mathrm{dL}^{-1}\right)$ & $14.1 \pm 1.2$ & $16.9 \pm 0.8^{*}$ & $<0.001$ & $14.4 \pm 0.8$ & $15.9 \pm 0.9^{*}$ & $<0.001$ & 0.371 & 0.002 \\
\hline $\mathrm{Hct}_{\text {peak }}(\%)$ & $43.1 \pm 3.7$ & $51.8 \pm 2.5^{*}$ & $<0.001$ & $44.1 \pm 2.3$ & $48.8 \pm 2.9^{*}$ & $<0.001$ & 0.382 & 0.003 \\
\hline \multicolumn{9}{|l|}{ Peak hemodynamics } \\
\hline $\mathrm{SBP}_{\text {peak }}(\mathrm{mm} \mathrm{Hg})$ & $174.9 \pm 33.2$ & $185.0 \pm 49.3$ & 0.587 & $227.1 \pm 52.3^{\mathrm{a}}$ & $231.1 \pm 48.5^{\mathrm{a}}$ & 0.785 & 0.005 & 0.018 \\
\hline $\mathrm{DBP}_{\text {peak }}(\mathrm{mm} \mathrm{Hg})$ & $115.9 \pm 40.9$ & $85.7 \pm 31.2$ & 0.075 & $114.2 \pm 29.4$ & $115.1 \pm 31.3^{\mathrm{a}}$ & 0.918 & 0.894 & 0.019 \\
\hline $\mathrm{MBP}_{\text {peak }}(\mathrm{mm} \mathrm{Hg})$ & $109.6 \pm 27.1$ & $111.0 \pm 35.1$ & 0.917 & $144.8 \pm 35.2^{\mathrm{a}}$ & $148.7 \pm 35.2^{\mathrm{a}}$ & 0.713 & 0.006 & 0.008 \\
\hline $\operatorname{SVR}_{\text {peak }}\left(\right.$ dyn s cm $\left.\mathrm{cm}^{-5}\right)$ & $810.1 \pm 272.3$ & $571.8 \pm 209.4^{*}$ & 0.038 & $1487.1 \pm 796.6^{\mathrm{a}}$ & $1004.7 \pm 268.2^{* a}$ & 0.010 & 0.010 & $<0.001$ \\
\hline $\mathrm{HR}_{\text {peak }}(\mathrm{bpm})$ & $168.8 \pm 9.9$ & $179.9 \pm 9.41^{*}$ & 0.005 & $146.4 \pm 12.3^{\mathrm{a}}$ & $144.5 \pm 13.5^{\mathrm{a}}$ & 0.586 & $<0.001$ & $<0.001$ \\
\hline \multicolumn{9}{|l|}{ Peak echocardiography } \\
\hline$R A_{\text {peak }}\left(m L m^{-2}\right)$ & $14.52 \pm 4.06$ & $13.31 \pm 4.04$ & 0.437 & $12.77 \pm 5.03$ & $15.99 \pm 7.55$ & 0.066 & 0.267 & 0.223 \\
\hline RV EDA $A_{\text {peak }}\left(\mathrm{cm}^{-2} \mathrm{~m}^{2}\right)$ & $9.50 \pm 1.72$ & $10.54 \pm 1.96$ & 0.146 & $8.76 \pm 1.85$ & $10.72 \pm 2.32^{*}$ & 0.001 & 0.222 & 0.801 \\
\hline RV ESA $A_{\text {peak }}\left(\mathrm{cm}^{2} \mathrm{~m}^{-2}\right)$ & $3.94 \pm 0.88$ & $4.31 \pm 0.90$ & 0.283 & $3.66 \pm 0.84$ & $4.14 \pm 1.44$ & 0.138 & 0.315 & 0.687 \\
\hline$L A_{\text {peak }}\left(m L I m^{-2}\right)$ & $13.82 \pm 5.08$ & $12.60 \pm 2.65$ & 0.433 & $17.53 \pm 6.83$ & $18.75 \pm 9.67^{\mathrm{a}}$ & 0.583 & 0.079 & 0.025 \\
\hline $\operatorname{LVEDV}_{\text {peak }}\left(\mathrm{mL} \mathrm{m}^{-2}\right)$ & $45.12 \pm 10.25$ & $54.10 \pm 9.84^{*}$ & 0.026 & $45.34 \pm 8.97$ & $55.23 \pm 14.76^{*}$ & 0.004 & 0.944 & 0.799 \\
\hline $\operatorname{LVESV}_{\text {peak }}\left(\mathrm{mL} \mathrm{m}^{-2}\right)$ & $8.24 \pm 2.10$ & $9.73 \pm 2.45$ & 0.095 & $9.80 \pm 3.53$ & $11.25 \pm 4.32$ & 0.170 & 0.135 & 0.232 \\
\hline LVEFpeak (\%) & $81.5 \pm 3.2$ & $81.51 \pm 4.92$ & 0.983 & $78.6 \pm 5.9$ & $79.6 \pm 6.1$ & 0.548 & 0.090 & 0.306 \\
\hline $\mathrm{SV}_{\text {peak }}\left(\mathrm{mL} \mathrm{m}^{-2}\right)$ & $36.90 \pm 8.98$ & $44.36 \pm 9.81^{*}$ & 0.046 & $35.54 \pm 7.20$ & $43.97 \pm 12.54^{*}$ & 0.003 & 0.595 & 0.921 \\
\hline$Q_{\text {peak }}\left(L \min ^{-1} \mathrm{~m}^{-2}\right)$ & $6.17 \pm 1.26$ & $7.99 \pm 1.86^{*}$ & 0.006 & $5.19 \pm 1.09^{\mathrm{a}}$ & $6.39 \pm 2.12^{* a}$ & 0.011 & 0.012 & 0.022 \\
\hline \multicolumn{9}{|l|}{ Peak pulmonary variables } \\
\hline $\mathrm{VO}_{2 \text { peak }}\left(\mathrm{mL} \mathrm{min}^{-1}\right)$ & $2699.7 \pm 552.4$ & $3826.9 \pm 829.6^{*}$ & $<0.001$ & $1818.0 \pm 498.8^{\mathrm{a}}$ & $2607.4 \pm 514.9^{* a}$ & $<0.001$ & $<0.001$ & $<0.001$ \\
\hline$V^{2 \text { peak }}\left(\mathrm{mL} \mathrm{min}^{-1} \mathrm{~kg}^{-1}\right)$ & $41.7 \pm 8.6$ & $49.5 \pm 11.5$ & 0.051 & $30.3 \pm 7.4^{\mathrm{a}}$ & $32.9 \pm 7.6^{\mathrm{a}}$ & 0.186 & $<0.001$ & $<0.001$ \\
\hline$V e_{\text {peak }}\left(L \min ^{-1}\right)$ & $56.8 \pm 28.9$ & $69.4 \pm 38.6$ & 0.337 & $58.3 \pm 21.5$ & $70.8 \pm 30.8$ & 0.081 & 0.857 & 0.900 \\
\hline
\end{tabular}

Significant $P$ values $(<0.05)$ are highlighted in bold

Data are expressed as mean \pm SD

$\mathrm{DBP}_{\text {peak' }}$, peak diastolic blood pressure; $\mathrm{Hb}_{\text {peak' }}$, peak hemoglobin concentration; $\mathrm{Hct}_{\text {peak' }}$, peak hematocrit; $\mathrm{HR}_{\text {peak' }}$ peak heart rate; $\mathrm{LA}_{\text {peak' }}$ peak left atria; $\mathrm{LVEDV}_{\text {peak' }}$ peak left ventricular end-diastolic volume; $\mathrm{LVEF}_{\text {peak, }}$ peak left ventricular ejection fraction; $\mathrm{MBP}_{\text {peak, }}$ peak mean blood pressure; $\mathrm{O}_{2}$, oxygen; $Q_{\text {peak, }}$ peak cardiac output; $\mathrm{RA}_{\text {peak }}$, peak right atria; $\mathrm{RV} E D A_{\text {peak }}$, peak right ventricular end-diastolic area; $\mathrm{RVESA}_{\text {peak }}$, peak right ventricular end-systolic area; $\mathrm{SBP}_{\text {peak' }}$, peak systolic blood pressure; $S V_{\text {peak }}$ peak stroke volume: $S R_{\text {peak }}$ peak systemic vascular resistance; $V_{2} O_{2 \text { peak }}$, peak oxygen uptake

${ }^{*} P<0.05$ compared with age-matched women

${ }^{\text {a }} P<0.05$ compared with sex-matched young individuals

at diastole were noted in older men compared with agematched women $(P \leq 0.005)$. Moreover, LVEDV $_{100 \mathrm{~W}}$ was elevated in both older and young men relative to age-matched women $(P \leq 0.049)$. A sex effect in $\mathrm{SV}_{100 \mathrm{~W}}$ reached significance in younger men, who presented higher $\mathrm{SV}_{100 \mathrm{w}}$ than age-matched women $(P=0.019)$. No effects of age were observed for submaximal cardiac structure and function $(P \geq 0.108)$. Concerning submaximal pulmonary variables, absolute $\mathrm{O}_{2}$ consumption was not affected by sex or age $(P \geq 0.535)$, whereas ventilation was augmented with older age in women $(P=0.033)$.

\section{Sex and age effects on cardiovascular volumetric relationships}

Figures 1 and 2 present the effects of sex and age, respectively, on fundamental volumetric relationships in the circulatory system, i.e., BV-LVEDV and LVEDVSV at peak exercise. The slopes of BV-LVEDV ${ }_{\text {peak }}$ and $\mathrm{LVEDV}_{\text {peak }}-\mathrm{SV}_{\text {peak }}$ did not differ according to sex in each age group $(P \geq 0.062)$, thus the analysis of the effect of sex on adjusted means was subsequently performed (Fig. 1). A unique sex effect was detected for the adjusted means analysis of BV-LVEDV $\mathrm{peak}_{\text {in }}$ older individuals, demonstrating a lower $L V E D V_{\text {peak }}$ for a given BV in women relative to men $(P=0.024)$. With respect to the effect of age, the slopes of BV-LVEDV $V_{\text {peak }}$ and $L V E D V_{\text {peak }}-S_{\text {peak }}$ were similar in young and older individuals, irrespective of sex $(P \geq 0.078)$ (Fig. 2). The subsequent analysis of adjusted means in each sex group did not detect age effects $(P \geq 0.087)$. 
Table 4 Hemodynamic, cardiac and pulmonary variables at a fixed submaximal workload (100 W)

\begin{tabular}{|c|c|c|c|c|c|c|c|c|}
\hline & \multicolumn{3}{|l|}{ Young } & \multicolumn{3}{|l|}{ Older } & \multicolumn{2}{|c|}{$\begin{array}{l}P \text { for age } \\
\text { comparison }\end{array}$} \\
\hline & q & $\hat{\sigma}$ & $P$ for sex & q & $\hat{0}$ & $P$ for sex & q & $\sigma^{2}$ \\
\hline \multicolumn{9}{|l|}{ Submaximal hemodynamics } \\
\hline $\mathrm{SBP}_{100 \mathrm{w}}(\mathrm{mm} \mathrm{Hg})$ & $159.0 \pm 21.2$ & $157.2 \pm 34.3$ & 0.878 & $218.6 \pm 48.9^{a}$ & $205.3 \pm 39.5^{a}$ & 0.318 & $<0.001$ & $<0.001$ \\
\hline $\mathrm{DBP}_{100 \mathrm{w}}(\mathrm{mm} \mathrm{Hg})$ & $96.6 \pm 27.3$ & $90.5 \pm 19.5$ & 0.536 & $111.4 \pm 24.9$ & $103.5 \pm 21.1$ & 0.257 & 0.124 & 0.084 \\
\hline $\mathrm{MBP}_{100 \mathrm{w}}(\mathrm{mm} \mathrm{Hg})$ & $107.4 \pm 13.8$ & $109.5 \pm 23.0$ & 0.791 & $143.2 \pm 31.3^{\mathrm{a}}$ & $135.0 \pm 24.9^{a}$ & 0.337 & $<0.001$ & 0.006 \\
\hline $\mathrm{SVR}_{100 \mathrm{~W}}\left(\right.$ dyn s cm $\left.\mathrm{cm}^{-5}\right)$ & $1137.7 \pm 246.7$ & $1011.6 \pm 310.1$ & 0.283 & $1923.5 \pm 712.6^{\mathrm{a}}$ & $1573.8 \pm 591.5^{\mathrm{a}}$ & 0.079 & $<0.001$ & 0.004 \\
\hline $\mathrm{HR}_{100 \mathrm{w}}(\mathrm{bpm})$ & $123.5 \pm 20.1$ & $105.6 \pm 14.9^{*}$ & 0.013 & $121.0 \pm 15.0$ & $102.2 \pm 11.3^{*}$ & $<0.001$ & 0.655 & 0.415 \\
\hline \multicolumn{9}{|l|}{ Submaximal echocardiography } \\
\hline $\mathrm{RA}_{100 \mathrm{~W}}\left(\mathrm{~mL} \mathrm{~m} \mathrm{~m}^{-2}\right)$ & $16.10 \pm 7.50$ & $16.74 \pm 5.56$ & 0.801 & $13.41 \pm 3.92$ & $17.49 \pm 6.01^{*}$ & 0.005 & 0.137 & 0.701 \\
\hline RVEDA ${ }_{100 \mathrm{w}}\left(\mathrm{cm}^{2} \mathrm{~m}^{-2}\right)$ & $10.05 \pm 2.77$ & $11.17 \pm 1.98$ & 0.233 & $9.07 \pm 2.05$ & $10.72 \pm 1.67^{*}$ & 0.002 & 0.207 & 0.451 \\
\hline $\operatorname{RVESA} A_{100 \mathrm{w}}\left(\mathrm{cm}^{2} \mathrm{~m}-{ }^{2}\right)$ & $4.42 \pm 1.40$ & $4.47 \pm 0.89$ & 0.910 & $3.77 \pm 1.10$ & $4.06 \pm 1.20$ & 0.345 & 0.108 & 0.266 \\
\hline $\mathrm{LA}_{100 \mathrm{w}}\left(\mathrm{mL} \mathrm{m} \mathrm{m}^{-2}\right)$ & $15.07 \pm 7.40$ & $15.53 \pm 7.18$ & 0.870 & $17.09 \pm 6.23$ & $19.98 \pm 7.89$ & 0.142 & 0.362 & 0.085 \\
\hline $\operatorname{LVEDV}_{100 \mathrm{w}}\left(\mathrm{mL} \mathrm{m}^{-2}\right)$ & $46.16 \pm 7.91$ & $56.43 \pm 9.32^{*}$ & 0.004 & $43.03 \pm 8.87$ & $49.87 \pm 15.19^{*}$ & 0.049 & 0.274 & 0.149 \\
\hline $\operatorname{LVESV}_{100 \mathrm{w}}\left(\mathrm{mL} \mathrm{m} \mathrm{m}^{-2}\right)$ & $10.22 \pm 2.39$ & $13.51 \pm 2.98^{*}$ & 0.003 & $10.05 \pm 3.99$ & $12.48 \pm 6.64$ & 0.108 & 0.882 & 0.586 \\
\hline $\operatorname{LVEF}_{100 \mathrm{~W}}(\%)$ & $77.9 \pm 3.4$ & $75.8 \pm 5.2$ & 0.229 & $77.1 \pm 6.1$ & $75.6 \pm 8.8$ & 0.466 & 0.662 & 0.918 \\
\hline $\mathrm{SV}_{100 \mathrm{~W}}\left(\mathrm{~mL} \mathrm{\textrm {m } ^ { - 2 }}\right)$ & $35.90 \pm 6.41$ & $42.91 \pm 8.36^{*}$ & 0.019 & $32.98 \pm 6.61$ & $37.39 \pm 11.62$ & 0.093 & 0.185 & 0.123 \\
\hline $\mathrm{Q}_{100 \mathrm{w}}\left(\mathrm{L} \mathrm{min}^{-1} \mathrm{~m}^{-2}\right)$ & $4.37 \pm 0.70$ & $4.51 \pm 0.92$ & 0.656 & $3.95 \pm 0.73$ & $3.85 \pm 1.37$ & 0.724 & 0.082 & 0.109 \\
\hline \multicolumn{9}{|c|}{ Submaximal pulmonary variables } \\
\hline $\mathrm{VO}_{2,100 \mathrm{~W}}\left(\mathrm{~mL} \mathrm{~min}^{-1}\right)$ & $1395.1 \pm 331.7$ & $1349.4 \pm 359.0$ & 0.760 & $1314.7 \pm 355.8$ & $1376.2 \pm 367.1$ & 0.535 & 0.524 & 0.838 \\
\hline 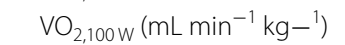 & $21.4 \pm 4.5$ & $17.1 \pm 4.0^{*}$ & 0.028 & $22.2 \pm 6.2$ & $17.5 \pm 5.1^{*}$ & 0.003 & 0.695 & 0.837 \\
\hline$V e_{100 w}\left(L_{m i n}^{-1}\right)$ & $21.9 \pm 9.0$ & $20.6 \pm 15.1$ & 0.801 & $33.0 \pm 15.4^{\mathrm{a}}$ & $28.8 \pm 13.9$ & 0.293 & 0.033 & 0.117 \\
\hline
\end{tabular}

Significant $P$ values $(<0.05)$ are highlighted in bold

Data are expressed as mean \pm SD

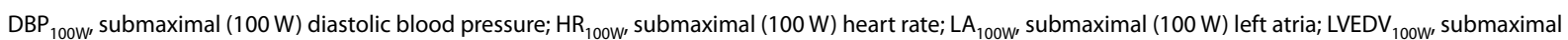
$(100 \mathrm{~W})$ left ventricular end-diastolic volume; $\mathrm{LVEF}_{100 \mathrm{w}}$, submaximal $\left(100 \mathrm{~W}\right.$ ) left ventricular ejection fraction; $\mathrm{MBP}_{100 \mathrm{w}}$, submaximal (100 W) mean blood pressure; $\mathrm{Q}_{100 \mathrm{~W}}$ submaximal $(100 \mathrm{~W})$ cardiac output; $\mathrm{RA}_{100 \mathrm{~W}^{\prime}}$ submaximal $(100 \mathrm{~W})$ right atria; $\mathrm{RV} \mathrm{EDA}_{100 \mathrm{w}}$, submaximal $(100 \mathrm{~W})$ right ventricular end-diastolic area; $\mathrm{RV}$ ESA $\mathrm{A}_{100 \mathrm{~W}}$ submaximal $(100 \mathrm{~W})$ right ventricular end-systolic area; $\mathrm{SBP}_{100 \mathrm{~W}}$, submaximal $(100 \mathrm{~W})$ systolic blood pressure; $\mathrm{SV}_{100 \mathrm{~W}}$, submaximal $(100 \mathrm{~W})$ stroke volume: $\mathrm{SVR}_{100 \mathrm{~W}}$ submaximal $(100 \mathrm{~W})$ systemic vascular resistance; $\mathrm{Ve}_{100 \mathrm{w}}$, submaximal $(100 \mathrm{~W})$ ventilation; $\mathrm{VO}_{2,100 \mathrm{~W}}$, submaximal $(100 \mathrm{~W})$ oxygen uptake

${ }^{*} P<0.05$ compared with age-matched women

a $P<0.05$ compared with sex-matched young individuals

\section{Discussion}

The main purpose of this study was to assess the influence of sex and age on key physiological determinants of cardiorespiratory fitness. To this end, hematological, cardiac, hemodynamic and $\mathrm{O}_{2}$ uptake variables at rest and during exercise were analyzed in a cohort of healthy young and older women and men presenting with similar levels of physical activity. The major findings are: (1) BV adjusted by body weight do not differ between sexes; (2) women present smaller LV dimensions and output at peak exercise compared with men, regardless of age and sex differences in body size; (3) for a given absolute BV, older women exhibit lower $\mathrm{LVEDV}_{\text {peak }}$ than age-matched men. The present findings, along with those of ancillary nature that are conjointly developed hereunder, denote the interactive effects of sex and age on the fundamental basis of the circulatory system at peak working aerobic capacity.
The capacity to deliver $\mathrm{O}_{2}$ through the circulatory system, i.e., the main determinant of cardiorespiratory fitness $[9,10,13]$, shows great quantitative variability in humans. Both genotypic and phenotypic variation contribute to the wide range of cardiac and aerobic capacities across the lifespan [9]. Beyond the manifest alterations induced by certain cardiovascular and hematological conditions $[17,21,22]$, sex, age and physical activity levels are well-known to independently modulate key limiting factors of the $\mathrm{O}_{2}$ transport chain [20]. In this respect, human experimental investigations have demonstrated that not all steps of the $\mathrm{O}_{2}$ cascade from the atmosphere to the systemic circulation have the same importance $[10,36]$. Lung structural and functional characteristics, which could in principle limit $\mathrm{O}_{2}$ transport, are overbuilt in relation to the potential delivery of $\mathrm{O}_{2}$ via the systemic circulation in healthy individuals exercising in normoxic conditions [37]. In men and presumably in women, 


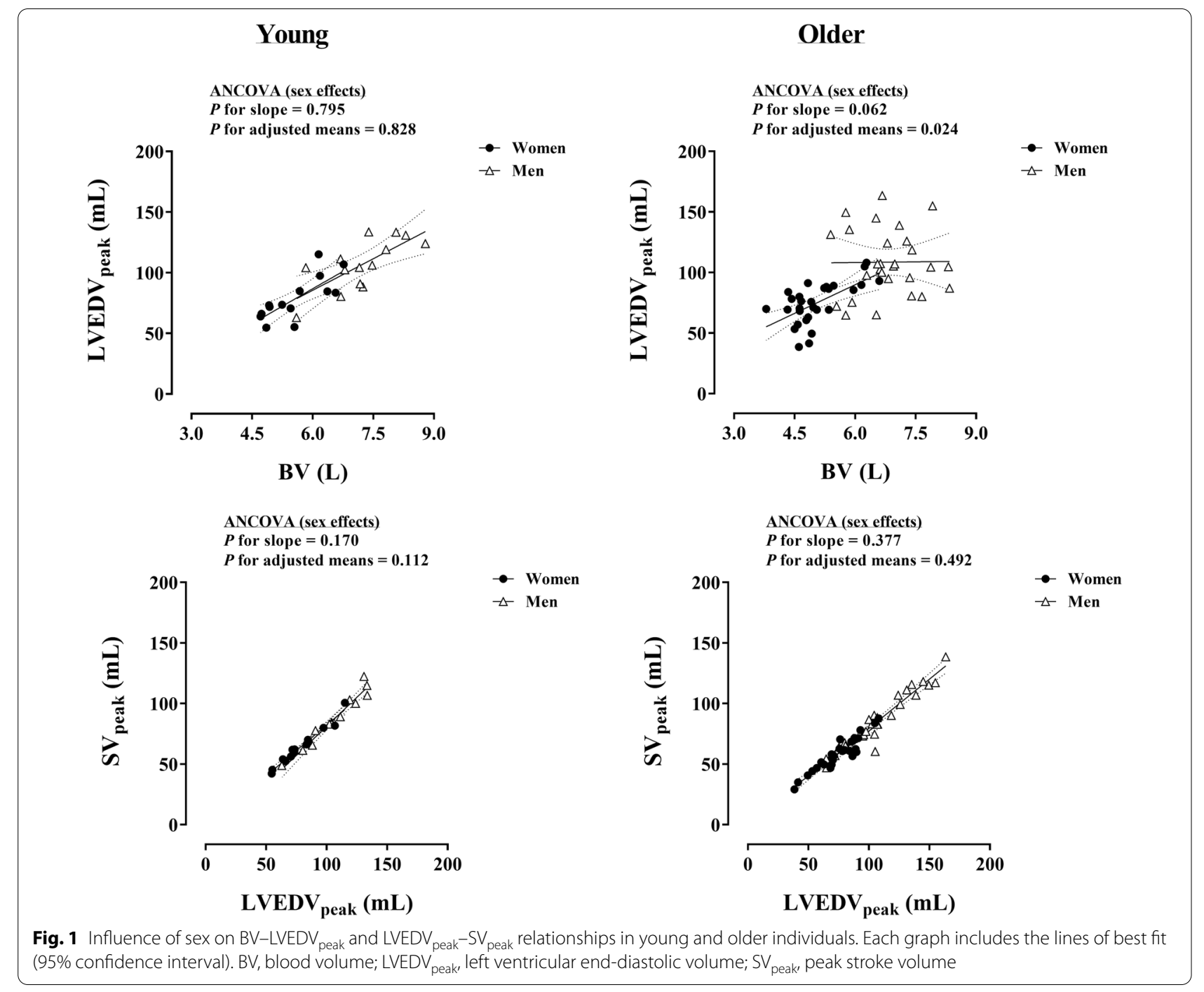

$\mathrm{VO}_{2 \text { peak }}$ is primarily a function of the heart's ability to pump blood and arterial $\mathrm{O}_{2}$ content $[9,10,13]$. The latter factor is determined by a relatively fixed and sex-specific hematological variable, i.e., blood $\mathrm{O}_{2}$ carrying capacity [24]. In contrast, cardiac pumping capacity is primarily subordinated to the filling level of the circulatory system, a rather modifiable variable $[6,13]$. The observation of similar BV per kg of body weight between sexes, notably in older individuals, yet substantially lower $S_{\text {peak }}$ in women versus men even after taking into account anthropometric differences suggests a potential novel sex dimorphism in a fundamental circulatory relationship [13-15]. In both young and older individuals, women exhibit nearly one-fifth lower SVpeak that is paralleled by a commensurate reduction in $L E D V_{\text {peak }}$ compared with men, i.e., the peak filling of the LV is substantially curtailed by the female sex. Integrative cardiovascular factors that may explain such a prominent sex dimorphism in the limitation of cardiorespiratory fitness are discussed hereunder.

The relationship between BV and $L V E D V_{\text {peak }}$ reflects the extent to which the distribution of blood is optimized within the circulatory system at peak working capacity. In older individuals, LVEDV $_{\text {peak }}$ per unit of BV was lower in women compared with men (Fig. 1). Sex or age effects were not observed in the relationship between $\mathrm{LVEDV}_{\text {peak }}$ and $\mathrm{SV}_{\text {peak }}$, indicating that the Frank-Starling mechanism was preserved. Thus, lower cardiac filling for a given BV may, at least in part, explain the reduced output $\left(Q_{\text {peak }}\right)$ in older women relative to age-matched men. Multiple cardiovascular regulatory mechanisms that primarily determine cardiorespiratory fitness might influence venous return and cardiac filling, ultimately limiting the systemic delivery of blood and thereby $\mathrm{O}_{2}$ [9]. These potential 'weakest' links can be in principle classified as belonging (intrinsic/central) or not (extrinsic/peripheral) 

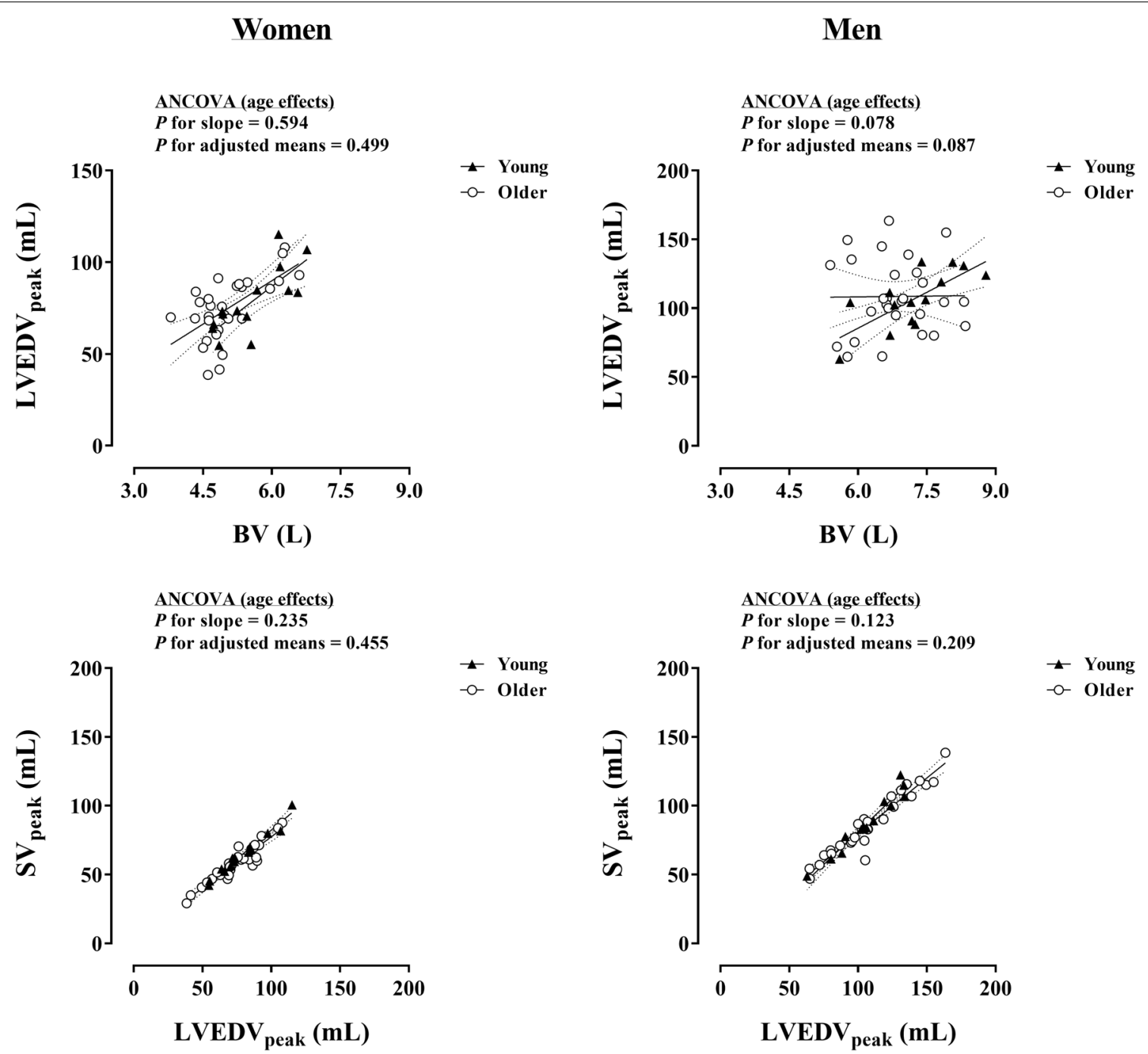

Fig. 2 Influence of age on BV-LVEDV $V_{\text {peak }}$ and LVEDV ${ }_{\text {peak }}-S V_{\text {peak }}$ relationships in women and men. Each graph includes the lines of best fit (95\% confidence interval). BV, blood volume; LVEDV peak $_{\text {I }}$ left ventricular end-diastolic volume; SV $V_{\text {peak, }}$ peak stroke volume

to the anatomy and physiology of the heart. With respect to central factors, it is tempting to hypothesize a role for women's predisposition to cardiac stiffness and impaired LV relaxation with advanced age [16, 21, 22, 38]. Women commonly present with augmented LV diastolic and systolic stiffness and steeper age-related increases in LV systolic stiffness compared with men [38]. Main LV functions may thus be prominently impaired in women with advance age, since older age per se (including men) did not alter the fundamental volumetric relationships (BV and LVEDV $_{\text {peak }}$, LVEDV the other hand, despite older age was associated with an impairment of common markers of LV diastolic function, they did not differ between sexes at rest (Table 1). Indeed, older women had similar mitral but higher tricuspid diastolic annular velocities at rest than age-matched men. While accurate non-invasive measures of diastolic function could not be obtained during exercise due time constraints for multiple cardiac imaging recordings at a given intensity, a prevailing association between resting and peak exercise diastolic function assessments has been found in catheterization studies [39, 40]. As regards peripheral factors, skeletal muscle arterial vasodilation is known to independently contribute to increase SV during exercise via the reduction of the systemic resistance to blood flow, i.e., SVR [41]. In this respect, we observed markedly augmented SVRpeak with female sex and older age. In addition, the myocardial isovolumic contraction time (IVCT) was increased with older age in women but not in men, which could also be partly related to 
female-specific LV systolic and central arterial stiffening [38]. Taken together, older women may have experienced the highest resistance to perfuse the systemic circulation at peak exercise, possibly resulting in lower output $\left(Q_{\text {peak }}\right)$, venous return and cardiac filling as a downstream consequence [42], which inverts the aforementioned causality sequence in the circulatory system [43]. Parenthetically, beyond the classic causality dilemma, whether a given factor within an intricate biological mechanism such as the circulatory system is conceptually considered as a cause or consequence depends on the standpoint. Experimental studies manipulating single variables at a time are needed to elucidate why older women exhibit higher $\mathrm{SVR}_{\text {peak }}$ and distribute their BV in a less optimal manner than opposite-sex counterparts. Such efforts might also shed light into resembling and highly prevalent pathophysiological alterations underlying deathly cardiac conditions in older women [21, 38, 44, 45].

A strong prognostic factor of cardiovascular health, $Q_{\text {peak }}$, was independently modulated by sex and age $[7,8]$. Specifically, both young and older women presented with reduced $Q_{\text {peak }}$ compared with men matched by age, physical activity levels as well as aerobic capacity. In fact, notwithstanding the relevance of cardiac pumping capacity as a hallmark of cardiorespiratory fitness, its pulmonary correlate, i.e., $\mathrm{VO}_{2 \text { peak }}$, did not differ between sexes. Likewise, despite cardiac output at a fixed submaximal workload $\left(Q_{100 \mathrm{w}}\right)$ was similar in women and men, submaximal $\mathrm{O}_{2}$ uptake $\left(\mathrm{VO}_{2100 \mathrm{~W}}\right)$ was higher in the former, regardless of age. Collectively considered, for a given unit of systemic blood flow $\left(Q_{\text {peak }}, Q_{100 w}\right)$, women extracted more $\mathrm{O}_{2}$ than men, albeit the female sex is typically characterized by reduced arterial $\mathrm{O}_{2}$ content owing to lower blood $\mathrm{O}_{2}$ carrying capacity throughout the adult lifespan [23], as herein demonstrated at rest and during exercise. The increased $\mathrm{VO}_{2} / \mathrm{Q}$ ratio in women may be underlined by central and peripheral factors. Starting from the periphery, recent studies have evidenced higher mitochondrial content and oxidative capacity, theoretically reflecting an enhanced potential for $\mathrm{O}_{2}$ extraction, in skeletal muscle fibers of women compared with men matched by $\mathrm{VO}_{2 \text { peak }}$ [46-48]. Furthermore, the current observation of higher $\mathrm{SVR}_{\text {peak }}$ in women could also reflect an increased tightening of the systemic circulation leading to improved blood flow distribution and enhanced $\mathrm{O}_{2}$ extraction at peak exercise [49]. Yet, at a fixed submaximal workload another factor could be at play. Women have smaller conducting airways than men, also when adjusted by lung size [50]. Consequently, the female lung's anatomy imposes greater mechanical resistance to breathing, particularly during exercise, thereby entailing a higher ventilatory work and $\mathrm{O}_{2}$ uptake (energy expenditure) for a given external work output [50]. The present findings therefore support the notion of women being less physiologically efficient than men, even when crucial counfounders are matched between sexes.

There are some limitations in this study that require comment. As an intrinsic limitation of cross-sectional analyses assessing the influence of sex and age, causeand-effect relationships cannot be established, thus the conclusions should be taken with caution. Very longterm (lifelong) longitudinal studies, while seldom implemented due to outstanding logistical and technical challenges [51], could eventually provide a stronger level of evidence. Moreover, we selected healthy individuals in order to limit the influence of disease-related confounding factors. Further research will elucidate whether the present findings can be extrapolated to specific clinical conditions.

\section{Perspectives and significance}

The current study assessed the impact of sex and age on major determinants of cardiorespiratory fitness in a cohort of healthy individuals matched by sex, age and physical activity levels. Following a comprehensive analysis at rest and during exercise, substantially smaller LV dimensions and correspondingly lower cardiac pumping capacity in women relative to men, independently of age and body size, are consistently demonstrated. Moreover, sex and age interact on a fundamental circulatory relationship, i.e., that of total circulating BV with LVEDV. Older women require higher BV for a certain level of LV filling, which suggests the presence of an intrinsic cardiovascular constraint, which concurs with functional features of prevalent life-threatening conditions in this population. Further experimental studies will ascertain whether such a circulatory limitation specific to older women can be overcome by tailored lifestyle and/or pharmacological interventions.

\section{Abbreviations}

BV: Blood volume; DBP: Diastolic blood pressure; $\mathrm{Hb}$ : Hemoglobin; $\mathrm{Hb}_{\text {mass: }}$ : Hemoglobin mass; Hct: Hematocrit; HR: Heart rate; LVEDV: Left ventricular enddiastolic volume; LVEF: Left ventricular ejection fraction; LVESV: Left ventricular end-systolic volume; MBP: Mean blood pressure; $\mathrm{O}_{2}$ : Oxygen; PV: Plasma volume; Q: Cardiac output; RBCV: Red blood cell volume; SBP: Systolic blood pressure; SV: Stroke volume; SVR: Systemic vascular resistance; $\mathrm{VO}_{2 \text { peak }}$ : Peak oxygen uptake.

\section{Supplementary Information}

The online version contains supplementary material available at https://doi. org/10.1186/s13293-021-00409-9.

Additional file 1: Fig. S1. Graphical outline of the study protocol. Fig. S2. Representative cardiac imaging during exercise according to the Simpson Method (biplane method of disks). 


\section{Acknowledgements}

The authors thank the study participants for their willingness, time and effort devoted to this study.

\section{Financial disclosure}

The authors have no financial relationships relevant to this article to disclose.

\section{Authors' contributions}

Conception and design of experiments: DM. Collection, analysis and interpretation: $C D, D M$. Drafting the article or revising it critically for important intellectual content: DM, CD. Both the author read and approved the final manuscript.

\section{Funding}

The project was funded by the Swiss National Science Foundation (P2ZHP3184211, to C.D.) and the Natural Sciences and Engineering Research Council of Canada (Discovery Grant, RGPIN-2019-04833, to D.M.).

\section{Availability of data and materials}

All data generated or analyzed during this study are included in the manuscript.

\section{Declarations}

\section{Ethics approval and consent to participate}

The study was approved by the Conjoint Health Research Ethics Board (REB181654) of the University of Calgary and conducted in accordance with the declaration of Helsinki. Prior to the start of the experiments, informed oral and written consents were obtained from all participants.

\section{Consent for publication}

Not applicable.

\section{Competing interests}

The authors have no conflicts of interest to disclose.

\section{Author details}

${ }^{1}$ Libin Cardiovascular Institute of Alberta, University of Calgary, Calgary, Canada. ${ }^{2}$ Cumming School of Medicine, Libin Cardiovascular Institute of Alberta, Faculty of Kinesiology, University of Calgary, 2500 University Drive NW, Calgary, AB, Canada. ${ }^{3}$ Faculty of Kinesiology, University of Calgary, Calgary, Canada.

\section{Received: 26 Auqust 2021 Accepted: 7 December 2021}

Published online: 03 January 2022

\section{References}

1. Covell JW, Ross J, Sonnenblick EH, Braunwald E. Comparison of the forcevelocity relation and the ventricular function curve as measures of the contractile state of the intact heart. Circ Res. 1966;19(2):364-72. https:// doi.org/10.1161/01.RES.19.2.364.

2. Patterson SW, Starling EH. On the mechanical factors which determine the output of the ventricles. J Physiol. 1914;48(5):357-79. https://doi.org/ 10.1113/jphysiol.1914.sp001669.

3. Maestrini D. Sulla genesi dell'automatismo cardiaco [About the origin of the automatism of the heart]. Arch di farmacologia sperimentale e scienze affini. 1915:467-80.

4. Bundgaard-Nielsen M, Sorensen H, Dalsgaard M, Rasmussen P, Secher NH. Relationship between stroke volume, cardiac output and filling of the heart during tilt. Acta Anaesthesiologica Scandinavica. 2009;53(10):13248. https://doi.org/10.1111/j.1399-6576.2009.02062.x.

5. Gregersen M, Rawson RA. Blood volume. Physiol Rev. 1959;39:307-42.

6. Kanstrup IL, Ekblom B. Acute hypervolemia, cardiac performance, and aerobic power during exercise. J Appl Physiol Resp Environ Exercise Physiol. 1982;52(5):1186-91. https://doi.org/10.1152/jappl.1982.52.5.1186.

7. Chomsky DB, Lang CC, Rayos GH, Shyr Y, Yeoh TK, Pierson RN 3rd, et al. Hemodynamic exercise testing. A valuable tool in the selection of cardiac transplantation candidates. Circulation. 1996;94(12):3176-83. https://doi. org/10.1161/01.cir.94.12.3176.
8. Metra M, Faggiano P, D'Aloia A, Nodari S, Gualeni A, Raccagni D, et al. Use of cardiopulmonary exercise testing with hemodynamic monitoring in the prognostic assessment of ambulatory patients with chronic heart failure. J Am Coll Cardiol. 1999;33(4):943-50. https://doi.org/10.1016/ s0735-1097(98)00672-x.

9. Lundby C, Montero D, Joyner M. Biology of VO2 max: looking under the physiology lamp. Acta Physiol (Oxf). 2017;220(2):218-28. https://doi.org/ 10.1111/apha.12827.

10. Montero D, Cathomen A, Jacobs RA, Fluck D, de Leur J, Keiser S, et al. Haematological rather than skeletal muscle adaptations contribute to the increase in peak oxygen uptake induced by moderate endurance training. J Physiol. 2015;593(20):4677-88. https://doi.org/10.1113/JP270250.

11. Panebianco RA, Stachenfeld N, Coplan NL, Gleim GW. Effects of blood donation on exercise performance in competitive cyclists. Am Heart J. 1995;130(4):838-40. https://doi.org/10.1016/0002-8703(95)90085-3.

12. Van Remoortel H, De Buck E, Compernolle V, Deldicque L, Vandekerckhove P. The effect of a standard whole blood donation on oxygen uptake and exercise capacity: a systematic review and meta-analysis. Transfusion. 2017;57(2):451-62. https://doi.org/10.1111/trf.13893.

13. Montero D, Lundby C. Regulation of red blood cell volume with exercise training. Compr Physiol. 2018;9(1):149-64. https://doi.org/10.1002/cphy. c180004.

14. Munch GD, Svendsen JH, Damsgaard R, Secher NH, Gonzalez-Alonso J, Mortensen SP. Maximal heart rate does not limit cardiovascular capacity in healthy humans: insight from right atrial pacing during maximal exercise. J Physiol. 2014;592(2):377-90. https://doi.org/10.1113/jphysiol.2013. 262246.

15. Guyton AC, Hall JE. Guyton and hall textbook of medical physiology. Philadelphia: Saunders; 2015.

16. Diaz-Canestro C, Montero D. The impact of sex on left ventricular cardiac adaptations to endurance training: a systematic review and meta-analysis. Sports Med. 2020;50(8):1501-13. https://doi.org/10.1007/ s40279-020-01294-9.

17. Georgieva Z, Georgieva M. Compensatory and adaptive changes in microcirculation and left ventricular function of patients with chronic iron-deficiency anaemia. Clin Hemorheol Microcirc. 1997;17(1):21-30.

18. Montero D, Diaz-Canestro C, Flammer A, Lundby C. Unexplained anemia in the elderly: potential role of arterial stiffness. Front Physiol. 2016;7:485. https://doi.org/10.3389/fphys.2016.00485.

19. Montero D, Lundby C. Red cell volume response to exercise training: association with aging. Scand J Med Sci Sports. 2017;27(7):674-83. https://doi.org/10.1111/sms.12798.

20. Ogawa T, Spina RJ, Martin WH 3rd, Kohrt WM, Schechtman KB, Holloszy $\mathrm{JO}$, et al. Effects of aging, sex, and physical training on cardiovascular responses to exercise. Circulation. 1992;86(2):494-503. https://doi.org/10. 1161/01.cir.86.2.494.

21. Diaz-Canestro C, Montero D. Female sex-specific curtailment of left ventricular volume and mass in HFpEF patients with high end-diastolic filling pressure. J Hum Hypertens. 2020;35(3):296-9. https://doi.org/10.1038/ s41371-020-00394-3.

22. Regitz-Zagrosek $V$, Kararigas $G$. Mechanistic pathways of sex differences in cardiovascular disease. Physiol Rev. 2017;97(1):1-37. https://doi.org/10. 1152/physrev.00021.2015.

23. Murphy WG, Tong E, Murphy C. Why do women have similar erythropoietin levels to men but lower hemoglobin levels? Blood. 2010;116(15):2861-2. https://doi.org/10.1182/blood-2010-07-294595.

24. Lundby C, Montero D. Did you know-why does maximal oxygen uptake increase in humans following endurance exercise training? Acta Physiol (Oxf). 2019;227(4):e13371. https://doi.org/10.1111/apha.13371.

25. Montero D, Diaz-Canestro C, Keiser S, Lundby C. Arterial stiffness is strongly and negatively associated with the total volume of red blood cells. Int J Cardiol. 2016;221:77-80. https://doi.org/10.1016/j.jjcard.2016. 06.265 .

26. Montero D, Houben AJ, Koster A, Muris DM, Schram MT, Gronenschild EH, et al. Physical activity is associated with glucose tolerance independent of microvascular function: the maastricht study. J Clin Endocrinol Metab. 2016;101(9):3324-32. https://doi.org/10.1210/jc.2016-1526.

27. Montero D, Breenfeldt-Andersen A, Oberholzer L, Haider T, Goetze JP, Meinild-Lundby AK, et al. Erythropoiesis with endurance training: dynamics and mechanisms. Am J Physiol Regul Integr Comp Physiol. 2017;312(6):R894-902. https://doi.org/10.1152/ajpregu.00012.2017. 
28. Siebenmann C, Keiser S, Robach P, Lundby C. CORP: the assessment of total hemoglobin mass by carbon monoxide rebreathing. J Appl Physiol (1985). 2017;123(3):645-54. https://doi.org/10.1152/japplphysiol.00185. 2017.

29. Lang RM, Badano LP, Mor-Avi V, Afilalo J, Armstrong A, Ernande L, et al. Recommendations for cardiac chamber quantification by echocardiography in adults: an update from the American Society of Echocardiography and the European Association of Cardiovascular Imaging. Eur Heart J Cardiovasc Imag. 2015;16(3):233-70. https://doi.org/10.1093/ehjci/jev014

30. Pellikka PA, Nagueh SF, Elhendy AA, Kuehl CA, Sawada SG, American Society of E. American Society of Echocardiography recommendations for performance, interpretation, and application of stress echocardiography. J Am Soc Echocardiogr. 2007;20(9):1021-41. https://doi.org/10.1016/j. echo.2007.07.003.

31. Du Bois D, Du Bois EF. A formula to estimate the approximate surface area if height and weight be known. Nutrition. 1989;5(5):303-11 (discussion 12-3)

32. Boda WL, Watenpaugh DE, Ballard RE, Hargens AR. Supine lower body negative pressure exercise simulates metabolic and kinetic features of upright exercise. J Appl Physiol (1985). 2000;89(2):649-54. https://doi.org/ 10.1152/jappl.2000.89.2.649.

33. Murthy G, Watenpaugh DE, Ballard RE, Hargens AR. Supine exercise during lower body negative pressure effectively simulates upright exercise in normal gravity. J Appl Physiol (1985). 1994;76(6):2742-8. https://doi.org/ 10.1152/jappl.1994.76.6.2742.

34. Nes BM, Janszky I, Wisloff U, Stoylen A, Karlsen T. Age-predicted maximal heart rate in healthy subjects: the HUNT fitness study. Scand J Med Sci Sports. 2013;23(6):697-704. https://doi.org/10.1111/j.1600-0838.2012. 01445.x.

35. Kaminsky LA, Imboden MT, Arena R, Myers J. Reference standards for cardiorespiratory fitness measured with cardiopulmonary exercise testing using cycle ergometry: data from the fitness registry and the importance of exercise national database (FRIEND) registry. Mayo Clin Proc. 2017;92(2):228-33. https://doi.org/10.1016/j.mayocp.2016.10.003.

36. Lundby C, Montero D. CrossTalk opposing view: diffusion limitation of $\mathrm{O} 2$ from microvessels into muscle does not contribute to the limitation of VO2 max. J Physiol. 2015;593(17):3759-61. https://doi.org/10.1113/JP270 550.

37. Dempsey JA, La Gerche A, Hull JH. Is the healthy respiratory system built just right, overbuilt, or underbuilt to meet the demands imposed by exercise? J Appl Physiol (1985). 2020;129(6):1235-56. https://doi.org/10.1152/ japplphysiol.00444.2020.

38. Redfield MM, Jacobsen SJ, Borlaug BA, Rodeheffer RJ, Kass DA. Age- and gender-related ventricular-vascular stiffening: a community-based study. Circulation. 2005;112(15):2254-62. https://doi.org/10.1161/CIRCULATIO NAHA. 105.541078.

39. Andersen MJ, Ersboll M, Bro-Jeppesen J, Gustafsson F, Hassager C, Kober $L$, et al. Exercise hemodynamics in patients with and without diastolic dysfunction and preserved ejection fraction after myocardial infarction. Circ Heart Fail. 2012;5(4):444-51. https://doi.org/10.1161/CIRCHEARTF AILURE.112.967919.

40. Peteiro J, Pazos P, Bouzas A, Pinon P, Estevez R, Castro-Beiras A. Assessment of diastolic function during exercise echocardiography: annulus mitral velocity or transmitral flow pattern? J Am Soc Echocardiogr. 2008;21(2):178-84. https://doi.org/10.1016/j.echo.2007.06.009.

41. Bada AA, Svendsen JH, Secher NH, Saltin B, Mortensen SP. Peripheral vasodilatation determines cardiac output in exercising humans: insight from atrial pacing. J Physiol. 2012;590(8):2051-60. https://doi.org/10. 1113/jphysiol.2011.225334.

42. Smith JR, Joyner MJ, Curry TB, Borlaug BA, Keller-Ross ML, Van Iterson EH, et al. Locomotor muscle group III/IV afferents constrain stroke volume and contribute to exercise intolerance in human heart failure. J Physiol. 2020;598(23):5379-90. https://doi.org/10.1113/JP280333.

43. Beard DA, Feigl EO. Understanding Guyton's venous return curves. Am J Physiol Heart Circ Physiol. 2011;301(3):H629-33. https://doi.org/10.1152/ ajpheart.00228.2011.

44. Lyle MA, Brozovich FV. HFpEF, a disease of the vasculature: a closer look at the other half. Mayo Clin Proc. 2018;93(9):1305-14. https://doi.org/10. 1016/j.mayocp.2018.05.001.
45. Montero D, Lundby C, Ruschitzka F, Flammer AJ. True anemia-red blood cell volume deficit-in heart failure: a systematic review. Circ Heart Fail. 2017;10:5. https://doi.org/10.1161/CIRCHEARTFAILURE.116.003610.

46. Montero D, Madsen K, Meinild-Lundby AK, Edin F, Lundby C. Sexual dimorphism of substrate utilization: differences in skeletal muscle mitochondrial volume density and function. Exp Physiol. 2018;103(6):851-9. https://doi.org/10.1113/EP087007.

47. Cardinale DA, Larsen FJ, Schiffer TA, Morales-Alamo D, Ekblom B, Calbet $J A L$, et al. Superior intrinsic mitochondrial respiration in women than in men. Front Physiol. 2018;9:1133. https://doi.org/10.3389/fphys.2018. 01133.

48. Diaz-Canestro C, Montero D. Unveiling women's powerhouse. Exp Physiol. 2020;105(7):1060-2. https://doi.org/10.1113/EP088395.

49. Calbet JA, Lundby C, Sander M, Robach P, Saltin B, Boushel R. Effects of ATP-induced leg vasodilation on $\mathrm{VO}_{2}$ peak and leg $\mathrm{O}_{2}$ extraction during maximal exercise in humans. Am J Physiol Regul Integr Comp Physiol. 2006;291(2):R447-53. https://doi.org/10.1152/ajpregu.00746.2005.

50. Sheel AW, Dominelli PB, Molgat-Seon Y. Revisiting dysanapsis: sex-based differences in airways and the mechanics of breathing during exercise. Exp Physiol. 2016;101(2):213-8. https://doi.org/10.1113/EP085366.

51. McGavock JM, Hastings JL, Snell PG, McGuire DK, Pacini EL, Levine BD, et al. A forty-year follow-up of the Dallas Bed Rest and Training study: the effect of age on the cardiovascular response to exercise in men. J Gerontol A Biol Sci Med Sci. 2009;64(2):293-9. https://doi.org/10.1093/gerona/ $g \ln 025$.

\section{Publisher's Note}

Springer Nature remains neutral with regard to jurisdictional claims in published maps and institutional affiliations.

Ready to submit your research? Choose BMC and benefit from:

- fast, convenient online submission

- thorough peer review by experienced researchers in your field

- rapid publication on acceptance

- support for research data, including large and complex data types

- gold Open Access which fosters wider collaboration and increased citations

- maximum visibility for your research: over $100 \mathrm{M}$ website views per year

At BMC, research is always in progress.

Learn more biomedcentral.com/submissions 